\title{
La evaluación de lenguas en un colegio plurilingüe: Un estudio de caso de las creencias pedagógicas del profesorado
}

\author{
Zita Mate \\ Universidad Autónoma de Barcelona, España
}

Artículo recibido 31/10/16, aceptado $14 / 02 / 17$, versión final 08/05/17

DOI: https://doi.org/10.5565/rev/jt13.724

\begin{abstract}
Resumen
El artículo describe los resultados de un estudio de caso que investiga las creencias, representaciones y saberes de profesores de lengua, indaga sobre cómo explican esos profesores sus prácticas de evaluación con alumnos que aprenden simultáneamente varias lenguas. Con esta finalidad, se escogió un centro internacional que lleva más de un siglo experimentando la enseñanza plurilingüe. Fueron realizadas tres entrevistas semiestructuradas cuyo análisis desveló que el docente de cada lengua entiende la evaluación como parte integrante del proceso de enseñanza-aprendizaje. Desde el punto de vista del alumno, los métodos de los docentes se complementan, aunque el planteamiento de la evaluación en cada caso es monolingüe.
\end{abstract}

Palabras clave: investigación de caso, evaluación, creencias pedagógicas, enseñanza de lenguas, plurilingüismo

\begin{abstract}
Resum
L'article descriu els resultats d'un estudi de cas que investiga les creences, representacions i sabers de professors de llengua, indaga sobre com expliquen aquests professors les seves pràctiques d'avaluació amb alumnes que aprenen simultàniament diverses llengües. Amb aquesta finalitat es va escollir un centre internacional que porta més d'un segle experimentant l'ensenyament plurilingüe. Es van realitzar tres entrevistes semi - estructurades, l'anàlisi de les quals desvelà que cada docent entén l'avaluació com a part integrant del procés d'ensenyament-aprenentatge. Des del punt de vista de l'alumne, els mètodes que empren els docents es complementen, encara que el plantejament de la seva avaluació en cada cas és monolingüe.
\end{abstract}

Paraules clau: investigació de cas, avaluació, creences pedagògiques, ensenyament de llengües, plurilingüisme,

\begin{abstract}
This article describes the results from a case study on the beliefs, representations and knowledge of language teachers, on how they explain their evaluation methods with students who simultaneously learn various languages. With this goal in mind, we chose an international school that has been experimenting with multilingual education for more than a century. Three semistructured interviews were carried out, the analysis of which revealed that all three teachers understand the evaluation as integrating key part of the educational process. From the point of view of the students, their teachers' methods complement each other even though the approach to evaluation in each case is monolingual.
\end{abstract}

Keywords: case study, evaluation, teacher's beliefs, language teaching, multilingualism 


\section{Introducción}

Este trabajo presenta una investigación de caso sobre las CRS (Creencias, Representaciones y Saberes, a continuación "creencias", Cambra, 2000) de profesores de diferentes lenguas acerca de la evaluación de alumnos plurilingües. Dado que la actividad evaluativa de los docentes es la que crea el marco en el que se desarrolla su labor, influye notablemente en la calidad de la misma y a la vez transmite valores a los alumnos tanto sobre el contenido como sobre la manera de aprender, parecía oportuno preguntarse cómo explican esta actividad los docentes. La investigación se llevó a cabo en un centro internacional que experimenta desde hace más de un siglo la enseñanza plurilingüe. Se diseñó un estudio de caso en el que se realizaron tres entrevistas semiestructuradas con un profesor de cada una de las lenguas vehiculares de este colegio excepto en el caso del catalán, donde prefirieron participar dos docentes juntos. Gracias al análisis de las entrevistas, previamente transcritas y traducidas, se pudieron reconstruir las creencias sobre la evaluación de los docentes de diferentes lenguas en el mismo centro con los mismos alumnos plurilingües.

Por tanto, el marco teórico de esta investigación aborda fundamentalmente tres campos: primero el de la evaluación, segundo el del plurilingüismo y tercero el de las creencias de los docentes.

\section{Evaluación}

Varios autores (Camps, 2003; Casanova, 1999; Cassany, Luna y Sanz, 2002) destacan la conveniencia de una 'evaluación formativa' en el proceso de aprendizaje:

La evaluación aplicada a la enseñanza y al aprendizaje consiste en un proceso sistemático y riguroso de recogida de datos, incorporado al proceso educativo desde su comienzo, de manera que sea posible disponer de información continua y significativa para conocer la situación, formar juicios de valor con respecto a ella y tomar decisiones adecuadas para proseguir la actividad educativa mejorándola progresivamente. (Casanova, 1999, p. 60)

Sin embargo, como llama la atención Lorente (2011), no se la puede confundir la evaluación formativa con la evaluación continuada, aunque a veces ambos términos se utilizan como sinónimos. No son comparables porque la evaluación continuada no incluye por sí misma una evaluación integrada en el proceso de enseñanza.

La evaluación debe ser 'formativa', es decir, formar parte integral del proceso de enseñanza-aprendizaje y su análisis debe tener valor informativo, tanto para el alumno como 
para el docente, con el fin de facilitar mejoras posteriores para ambos (Cassany, Luna y Sanz 2002; Ribas, 1997, 2010; Lorente 2011).

Esta evaluación, que comparte los criterios propios de la evaluación de los docentes, genera un cambio en las relaciones entre el docente y el alumno ya que, al prescindir del ánimo de juzgar y de la orientación hacia la selección, goza de la posibilidad de que los alumnos mismos puedan autoevaluarse o puedan coevaluar (heterocorreción) los trabajos de sus compañeros.

Este marco de la evaluación formativa es apto para acompañar la enseñanza de la escritura entendida como 'acto de la composición' (Camps, 2003), que es un proceso con diferentes etapas -la planificación, la redacción y la revisión-, y que requiere la adquisición de habilidades y micro habilidades lingüísticas y cognitivas, imprescindibles para la producción de un texto.

La sobrevaloración de la escritura en la enseñanza de lenguas contribuye a que la lengua oral tenga menos valor en ella (Cros y Vilá, 1997). Resulta que el peso de la enseñanza de la lengua oral depende también de las creencias del docente, así como de las características del centro o de los grupos de alumnos (Palou, 2008).

\section{Plurilingüismo}

En un mundo marcado por la diversidad, la fragmentación y la provisionalidad (Kress, 2013) se ha producido un aumento tanto de la sensibilidad hacía la diversidad lingüística como de la conciencia sobre la variedad de las lenguas (Franceschini, 2011). Y al mismo tiempo en cualquier aula hay cada vez más diferencias en las características lingüísticas de los alumnos con distintas procedencias. Hasta hace poco la única garantía de la enseñanza de las diferentes lenguas parecía ser la estricta separación de los idiomas de un hablante plurilingüe en las aulas (Jessner, 2008), o sea la multiplicación del monolingüismo (Lüdi y Py, 2009). Además, el objetivo tradicional de la enseñanza de las lenguas ha sido el aprendizaje de la lengua y del conocimiento de su patrimonio cultural. No obstante, hoy en día son mucho más requeridos los conocimientos de estrategias lingüístico - comunicativas para convertirse en un hablante plurilingüe con competencia intercultural (Luise, 2013).

Los hablantes plurilingües se distinguen de sus compañeros monolingües, como observó Bialystok (2009), por el uso de un vocabulario menos amplio que los primeros y porque su fluidez es algo menor. Sin embargo, demuestran una mayor flexibilidad cognitiva y una habilidad para un cambio más rápido de tareas. También presentan una mayor capacidad 
metalingüística para la solución de problemas, pero sobre todo destaca su dominio de la comunicación no verbal. Bialystok (2009) también dice que la competencia metalingüística de los alumnos bilingües es más alta que la de sus compañeros monolingües ya que distinguen mejor entre el sistema gramatical y el semántico, aunque les cuesta compararlos entre las diferentes lenguas. Guasch (2014) afirma que las competencias de la escritura son transversales y por este motivo los alumnos no deben aprender en cada lengua la estrategia completa sino solamente sus peculiaridades, sobre todo en el caso de la gramática y de la ortografía. Camps, Guasch, Milian, y Ribas (2005) mencionan la enseñanza de la segunda lengua como posible causa de problemas ortográficos. Galambos y Goldin-Meadow (1990) demostraron que los bilingües son al mismo tiempo más propensos a cometer errores, como a poder corregirlos, aunque sin saber dar explicaciones de sus causas. Guasch (2014) afirmó que los monolingües demuestran más sensibilidad hacia la gramaticalidad mientras los bilingües hacia la coherencia semántica. Sin embargo, en ningún caso se puede afirmar que uno de los grupos sea mejor o peor que el otro.

Blommaert y Backus (2012) subrayan que el término aprendizaje es el que describe el proceso de los plurilingües por su reversibilidad, ya que unos efectos del aprendizaje pueden ser más estables que otros y además en su caso tampoco supone el dominio completo del 'repertorio lingüístico' en todas las lenguas utilizadas por el hablante plurilingüe. El dinamismo de este proceso de aprendizaje consiste en la influencia mutua entre todas las lenguas aprendidas, es decir contradiciendo a Cook, quien supuso un influjo de la L1 hacia la L2, ahora sabemos que la L2 afecta también a la L1 (Kecskés y Papp, 2000), y en el caso de que haya más lenguas, existen impactos entre todas ellas y en todas las direcciones (Cenoz y Genesee, 1998; Guasch, 2014,). Del mismo modo como afirma la teoría del modelo dinámico de plurilingüismo elaborado por Herdina y Jessner (2002), todas las lenguas que conoce un hablante están interactuando unas con otras creando una transversalidad entre ellas. Y, además, la experiencia durante del proceso del aprendizaje de las dos primeras lenguas, especialmente gracias al desarrollo de la competencia estratégica, influye positivamente en el aprendizaje de las siguientes. Cinco son los factores responsables de este fenómeno (Hufeisen y Marx, 2007): a) Factores neurofisiológicos; b) Factores externos, como: socioculturales, socioeconómicos, tradición de aprendizaje, etc.; c) Factores emocionales; d) Factores cognitivos: conciencia lingüística, metalingüística y de aprendizaje, conocimiento de estilos de estudio y habilidades para aplicar estrategias de aprendizaje y e) Factores lingüísticos. 
El modelo de proceso plurilingüe de Meißner (2004) describe la construcción de una gramática espontánea por parte del alumno solo si hay una relación etimológica entre las lenguas, si el alumno dispone de un buen conocimiento de una lengua emparentada que actúe como "lengua puente" y facilite la "intercomprensión" del nuevo idioma, y si dispone de una estrategia de aprendizaje de la lengua. Ya que como afirma Jessner (2008) que el alumno de L3 se distingue del alumno de L2 porque en el caso de la adquisición de la L3, las estrategias propias del hablante ya están presentes, debido al hecho de haber logrado aprender la L2, ya ha desarrollado destrezas de aprendizaje, como por ejemplo la habilidad de comparar o de transferir. Durante este proceso ocurre que los alumnos al disponer ya de estrategias y experiencias de aprendizaje y empujados por estrategias comunicativas mejor desarrolladas cometen más errores que los monolingües y los que están aprendiendo su primera lengua extranjera, justamente por su actuación proactiva y arriesgada (Björklund, 2005). Y en este sentido, estos mismos errores incluyen su propia solución ya que son manifestaciones de un paso cognitivo inicial, aunque hayan terminado en un punto equivocado. No obstante, Cassany, Luna y Sanz (2002) subrayan que la falta de intervención en los errores puede conducir a la fosilización de los mismos en la segunda lengua. Sobre las dificultades en la evaluación de los plurilingües por error en el diagnóstico, a las que hacen referencia Bedore y Peña (2008) y Jessner (2008), llama la atención que la educación plurilingüe va a mejorar solo si las normas pedagógicas se orientan hacia el plurilingüismo (García y Kleyn, 2013).

\section{La evaluación de los alumnos plurilingües}

Lorenzo, Trujillo y Vez (2011) afirman que la evaluación es uno de los factores que intervienen en la calidad de los programas plurilingües. Sin embargo, hay que destacar sobre el monolingüismo y el plurilingüismo lo que ya mencionamos anteriormente, que la relación entre los dos no es jerárquica sino que son dos fenómenos diferentes y ninguno de los dos se puede medir desde el punto de vista del otro (Noguerol, 2001; Cambra, 2011; Nussbaum, 2014). Voet Cornelli, Schulz, y Tracy (2013) señalan que en el caso de alumnos plurilingües existe el peligro de estimar a la baja o al alza sus competencias lingüísticas. Se suele examinar la pronunciación, la fluidez, la competencia de expresión verbal y comprensión. En el caso de los monolingües el retraso del habla y el uso de frases con más elementos y además la aplicación de una gramática no correcta indican trastorno, mientras que los mismos síntomas pueden formar parte de un proceso correcto de aprendizaje de una persona plurilingüe. Por esta coincidencia de síntomas, puede darse el caso de que niños plurilingües con un desarrollo 
correcto suspendan la prueba del trastorno específico del desarrollo del lenguaje (TEDL) (mistaken identity) mientras que, por el contrario, otros alumnos plurilingües con el trastorno específico del desarrollo del lenguaje pueden pasar desapercibidos (missed identity; ver Welling, 1998; Rothweiler, 2011).

\section{Las creencias pedagógicas de los docentes}

El término de BAK (Beliefs, Assumption and Knowledge) fue creado por Woods (1996) y luego fue retomado por Cambra (2000), quien a través de su definición del término Creencias, Representaciones y Saberes (CRS) interrelaciona las creencias personales no estructuradas con las representaciones, que son enunciados cognitivos con dimensión social, pero poco estructurados, y con los saberes que se relacionan con el proceso de enseñanza/aprendizaje. Las creencias se hallan detrás del comportamiento de los docentes y son decisivas puesto que ejercen un efecto sobre los alumnos en el proceso del aprendizaje (Fives y Buehl, 2013). Aunque el nivel de coincidencia entre las creencias y la actuación es esencial para la eficacia del docente (Fives y Buehl, 2013), las discrepancias o tensiones entre ellas pueden contribuir al progreso del pensamiento del mismo (Birello y Sánchez-Quintana, 2013).

Fives y Buehl (2013) distinguen siete tipos de creencias del profesorado: 1) las que se refieren a sí mismo; 2) las que se refieren al contexto y al entorno; 3) las que se refieren al material escolar y al saber; 4) las que se refieren a la didáctica; 5) las que se refieren al marco metodológico de la enseñanza 6) las que se refieren a los alumnos y 7) la relación de las creencias de los docentes con sus prácticas educativas.

Las creencias dependen tanto de factores internos como externos (Fives y Buehl, 2013; Birello y Sánchez Quintana, 2013). Entre los factores internos destacan el conocimiento sobre la didáctica y la autoestima del docente, o sea la propia convicción sobre su eficacia. Los factores externos son más variados. Incluyen los pretextos culturales, la pertenencia a un barrio, región, país, etc., la cultura del colegio mismo, así como las clases, los alumnos o los padres de estos.

Las creencias actúan en tres niveles diferentes (Fives y Buehl, 2013). Primero, pueden proceder como un filtro influyendo en la interpretación de la información durante las fases de la formación y la experiencia, o sea la interpretación de situaciones escolares incluso la toma de decisiones sobre el material para enseñar y en cómo enseñarlo. Segundo, las creencias pueden formar un marco que lleva al docente a la elaboración de su concepto educativo desde 
lo que considera como su tarea de profesor hasta la creación de sus planes de clase o sus reflexiones sobre la misma. Y tercero, las creencias incluso pueden acompañar como una guía al docente para afinar los ejercicios concretos en sus clases y brindarle la automotivación y la convicción sobre el éxito de su propia labor (Fives y Buehl, 2013). Phipps y Borg (2009) aconsejan que los profesores exploren y reflexionen sobre sus creencias y las contrasten con sus prácticas para tomar conciencia de las relaciones entre ellas.

\section{Las creencias sobre el plurilingüismo}

La mitificación negativa del plurilingüismo es una historia que nos acompaña desde el siglo XVIII, cuando en Europa fueron impuestas las lenguas nacionales, y se ha extendido hasta los años 90 del pasado siglo, generando una relación ambigua entre las ambiciones y los rechazos o miedos. Se creía que el plurilingüismo conduciría a la pérdida de la lengua y que podría tener impactos negativos en el desarrollo de la personalidad. No solo algunos lingüistas, sino incluso médicos, desaconsejaron la educación en varias lenguas, amenazando que esta podía causar problemas patológicos, como la tartamudez u otros trastornos lingüísticos (Welling, 1998). Estos prejuicios fueron descartados y cada vez más científicos están demostrando que detrás de los trastornos del lenguaje hay una tendencia individual independiente de ser monolingüe o plurilingüe, o a veces ahí se esconden los miedos generados por un entorno negativo. Estos fenómenos son los que perjudican a una persona causándole dificultades. Ya que también la actitud hacia la nueva lengua o hacia las lenguas utilizadas es un factor importante. También influye la actitud de los docentes hacia los bilingües (Guasch, 2014).

\section{El objetivo y las preguntas de investigación}

El objetivo de esta investigación ha sido explorar las creencias pedagógicas de varios docentes que trabajan en un entorno plurilingüe (un colegio con tres idiomas vehiculares: catalán, castellano y alemán).

Para ello planteamos las siguientes preguntas:

¿Qué creen los profesores sobre su propio papel, sobre su entorno escolar y sobre sus alumnos plurilingües?

¿Cómo creen que debe ser la evaluación de lengua de alumnos plurilingües?

\section{El centro escolar}

La investigación se ha llevado a cabo en un centro plurilingüe que cuenta con una experiencia 
pedagógica de más de un siglo. En la actualidad se enseñan tres lenguas vehiculares en clases integradas por alumnos hablantes de L1, L2 y L3 en cada lengua respectivamente. El catalán y el castellano son lenguas ambientales y cooficiales en la región estando el castellano más implementado que el catalán. El alemán es lengua extranjera en el país. A parte de las tres lenguas vehiculares, se estudia el inglés como lengua extranjera (obligatorio para todos los alumnos) y el francés que puede sustituir el catalán a partir de secundaria. Existe también la posibilidad de mantener los cinco idiomas hasta el último curso, el nivel 12, que se cursa con 18 años. Los profesores de lenguas vehiculares son todos nativos en la suya y en muchos casos desconocen o conocen sólo parcialmente las otras lenguas. Sin embargo, todo el alumnado es o será plurilingüe, unos van convirtiéndose durante su trayectoria escolar, otros tienen ya a partir de su entrada al colegio un dominio de las lenguas propias del mismo e incluso algunos hablan además otras lenguas cuya enseñanza este centro no imparte.

El colegio sin embargo utiliza un sistema de clasificación de los alumnos que no les permite pertenecer a dos categorías. Si el alemán es su L1, son clasificados como alumnos de L2 en castellano, y si son alumnos de L2 en alemán, entonces obligatoriamente se suponen que su L1 es el castellano. En el caso del catalán, pueden asistir a clases de nivel avanzado (L1-L2) o para principiantes (L2-L3), dependiendo del criterio de los docentes.

En el colegio los tres departamentos de lengua trabajan independientemente, cumpliendo cada uno los requisitos del plan de estudios dictados por su ministerio de cultura correspondiente. Sin embargo, en la regulación de la evaluación existe un cierto reconocimiento mutuo que consiste en la valoración diferenciada de los alumnos dependiendo de la L1 que el colegio les asigna. Concretamente, utilizan unos baremos para evaluar trabajos escritos en los cuales los errores gramaticales se calculan más estrictamente en el caso de los alumnos reconocidos de L1 que en los de L2. Es decir, cada alumno será evaluado más estrictamente en su L1 que en su L2. Aquellos alumnos que entran al colegio con 10 años, en el $5^{\circ}$ curso, contarán con una evaluación todavía más permisiva de la lengua alemana después de una enseñanza en clases separadas durante tres años, a la que sigue su integración a partir del curso $8^{\circ}$ en clases de lenguas comunes con aquellos que llevan en el mismo centro desde la edad de 3 años.

\section{Metodología de la investigación}

El marco metodológico de esta investigación es la etnografía educativa y como instrumento se han utilizado entrevistas semiestructuradas (Sandín Esteban, 2003) y realizadas, utilizando en 
cada caso las mismas preguntas sobre la evaluación, sin mencionar las creencias y sin ideas preconcebidas, con los profesores. Las entrevistas se han usado posteriormente para describir, explicar e interpretar cualitativamente las creencias de estos docentes sobre la evaluación de alumnos plurilingües.

Con este fin se grabaron tres entrevistas, una con un docente de alemán, otra con uno de castellano y otra con dos docentes de catalán ya que estos prefirieron opinar conjuntamente. Esta última se realizó con dos sesiones, de una hora cada una y para las otras dos dedicamos a cada una de ellas una sesión de una hora. En cada entrevista semiestructurada fueron formuladas las mismas preguntas sobre la evaluación.

Todos estos profesores llevan más de una década en este centro educativo plurilingüe y gozan de un elevado reconocimiento profesional tanto entre los colegas como entre los alumnos.

\section{Análisis de los datos}

Las tres entrevistas fueron analizadas en la lengua original de la grabación. El análisis se realizó en cuatro fases siguiendo los procedimientos de Palou (2008).

En primer lugar se localizaron los fragmentos en los que los docentes, para justificar su actuación, iban explicando sus creencias sobre la evaluación de lengua con especial atención al plurilingüismo.

En segundo lugar las secuencias se clasificaron temáticamente. Se encontraron cuatro de las siete categorías que proponen Fives y Buehl (2013), que se refieren a (I.) su papel como docente, (II.), su entorno escolar, (III.) la evaluación y (IV.) los alumnos plurilingües. Luego fueron ordenadas en orden cronológico de aparición en el discurso para que cobraran más visibilidad las discrepancias o las creencias fijas de los docentes, expresadas en varias secuencias sobre el mismo aspecto. Los criterios de la transcripción de las entrevistas siguieron los utilizados por Palou (2008). ${ }^{\mathrm{i}}$

En tercer lugar, se analizó el modo en que el entrevistado explicaba su experiencia observando elementos como la utilización de pronombres personales o estructuras impersonales que muestran el posicionamiento del sujeto respecto a lo narrado, el tipo y el uso de conectores y marcadores para poder observar la relación entre los enunciados, los modos de verbo y perífrasis verbales, metáforas, elementos léxicos valorativos (Birello y Sánchez Quintana, 2013), y los énfasis o pausas en el habla para poder interpretar luego los textos con más exactitud. 


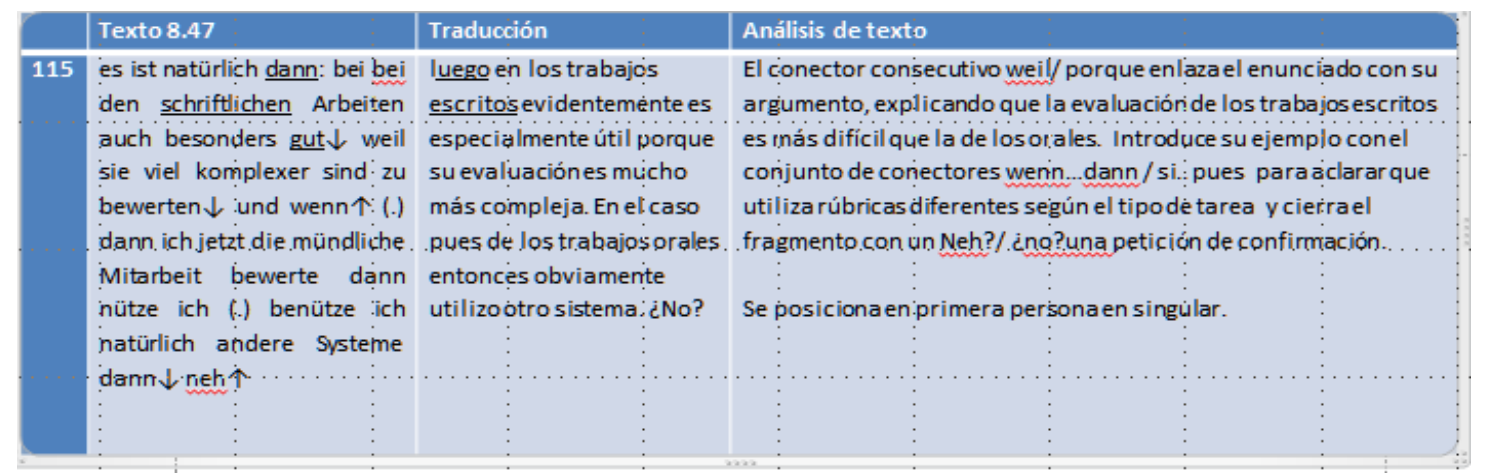

Fig. 1. Análisis con traducción ${ }^{\mathrm{ii}}$

A continuación se creó a través de las palabras clave de los profesores un mapa conceptual de cada una de las entrevistas para visualizar el conjunto de las creencias.

Por último, se hizo una comparación de los análisis de las tres entrevistas, prestando especial atención a los aspectos de la evaluación del plurilingüismo, para obtener una imagen integrada dentro del conjunto del sistema de este centro.

\section{Síntesis de los resultados}

En el análisis de las entrevistas sobre la evaluación hemos esclarecido cómo se ven los docentes a sí mismos, qué piensan sobre su estrategia de evaluación, sobre el colegio y sobre sus alumnos dentro del marco del plurilingüismo.

La normativa del colegio sobre la evaluación es muy detallada y evalúa con baremos diferentes a los alumnos de L1 y L2, permitiendo también aplicar un bonus iii en casos particulares, es decir se aplica una evaluación diferenciada (Noguerol, 2001; Cambra, 2011; Nussbaum, 2014). Dicha normativa exige además que haya un cierto equilibrio entre la evaluación del rendimiento escrito y del oral, aunque desde una perspectiva monolingüe que, por tanto, solo en parte se corresponde con la idea de García y Kleyn (2013) sobre las necesidades evaluativas de los plurilingües. Sin embargo, los docentes trabajan con tanta autonomía que pueden aplicar las normas exigidas por el centro según su criterio. Esto hizo posible que no se percibiera ninguna brecha entre su actuación (Birello y Sánchez Quintana, 2013) y su creencia, ya que cada uno pudo hacer un uso propio de la normativa y de este modo creó su propio sistema de evaluación mostrando características completamente diferentes en los tres casos. A través del análisis de sus razonamientos y de las explicaciones de sus actuaciones, que se reforzaron mutuamente, se pudo observar con más claridad las creencias que causaron el desarrollo de estas estrategias evaluativas propias. 
Los cuatro docentes entienden la evaluación como parte integral de la enseñanza y del aprendizaje de los alumnos (Casanova, 1999), y todos desarrollaron una estrategia de evaluación sistemática y compleja que incluye exigir a sus estudiantes que trabajen reflexionando sobre sus errores, para que sean ellos mismos los que se esfuercen para llegar a niveles de L1. Todos creen que el camino de aprendizaje es propio y diferente para cada alumno (Herdina y Jessner, 2002).

\section{El caso del profesor de alemán}

No obstante, el profesor alemán ve imprescindible el uso de herramientas evaluativas, tales como rúbricas, como se puede observar en el Fragmento 1, con el fin de que los alumnos puedan corregir mejor sus faltas. Le parece fundamental la existencia de un sistema de evaluación estructurado y diferenciado que tenga en cuenta las dificultades de los alumnos de L2 en un colegio con alumnos cuya L1 sea diferente de las lenguas del centro lo que se corresponde con lo que dice Cassany (2002). No habla en ningún momento sobre alumnos plurilingües, como tampoco sus colegas, sino que acepta la clasificación del colegio que distingue solamente entre dos grupos diferentes, es decir L1 y L2.

Fragmento 1:

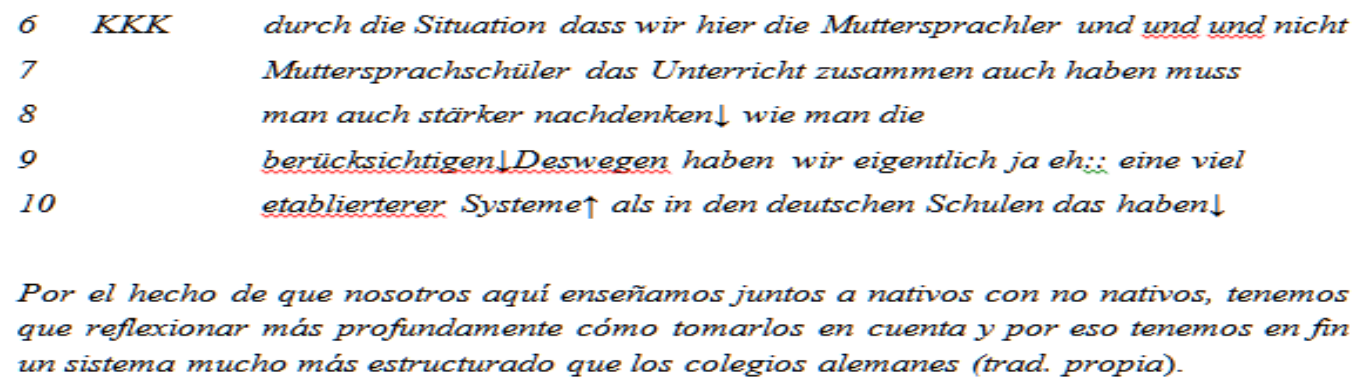

Tampoco habla mucho de las características de los alumnos descritas por Jessner (2008) sino más bien de los resultados de sus tareas. Aunque está convencido que ellos están de acuerdo con su método y lo valoran a pesar de que pueda perjudicarlos a la hora de obtener puntos, lo ven justo y entienden que con ello se refleja mejor su propio desarrollo, como se puede ver en el Fragmento 2: 
Fragmento 2:

\begin{tabular}{|c|c|c|}
\hline 762 & $K K K$ & im Endfall ist es auch wichtig $\uparrow$ das die \\
\hline 763 & & Schüler dieses Bewertungssistem dann ganz kla:r finden $\uparrow$ und es \\
\hline 764 & & akzeptieren $\uparrow$ und ich staune auch dass die Schüler eigentlich(..) neh:.: \\
\hline 765 & & ich staune nicht aber man könnte darüber staunen $\uparrow h: ;$ dass die \\
\hline 766 & & Schüler solche solche Bewertungssysteme auch sehr eh:: sehr schnell \\
\hline 767 & & akzeptieren $\downarrow$ \\
\hline
\end{tabular}

A fin de cuentas, es importante que a los alumnos les parezca muy claro el sistema de evaluación y que lo acepten. Me sorprende a mí también que los alumnos en el fondo..., no me sorprende, pero deberíamos sorprendernos de lo rápido que aceptan los alumnos tales sistemas de evaluación (trad. propia).

Este profesor cuenta con una amplia gama de herramientas evaluadoras más allá de los baremos impuestos por el colegio. Utiliza rúbricas diferentes según el tipo de tarea y además permite a los alumnos usar métodos diversos para que puedan trabajar con autonomía en la revisión y la corrección posterior. Sus criterios de evaluación además le sirven para afinar sus instrucciones y cerrar el círculo, lo que Casanova (1999) describe como la necesidad de concienciar más profundamente a los alumnos sobre su propio proceso de aprendizaje.

Dentro de su sistema, las notas cobran relevancia no solamente para que los alumnos puedan reflexionar sobre su propio desarrollo como propone Cassany (2002), sino para que estas sean también un certificado oficial del nivel alcanzado para los padres, el colegio o las autoridades y sirvan para una comparación que dé información para introducir nuevas estrategias. Aunque utiliza también las notas para motivar a los alumnos, p.ej. en el caso de tareas de menor importancia académica, dándoles mejores puntuaciones y permitiéndoles además que ellos colaboren en su propia evaluación.

Fragmento 3:

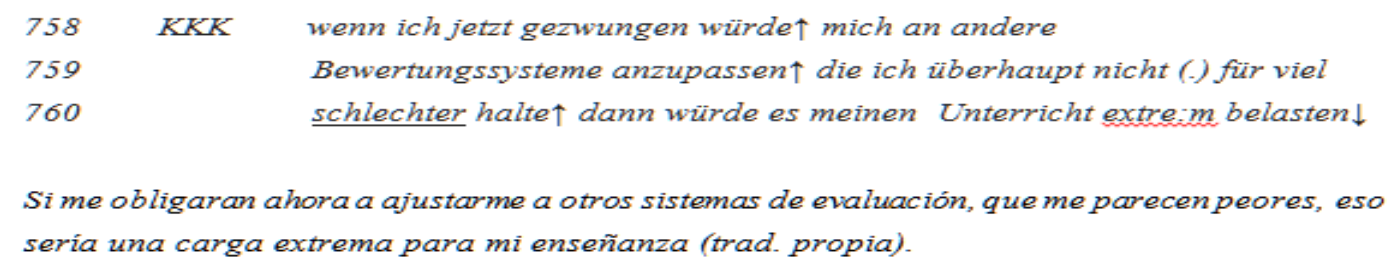

Defiende que cada docente pueda tener su propia estrategia de evaluación como se observa en el Fragmento 3 y que esta no sea impuesta por las autoridades. Sobre este punto, está de acuerdo con el docente de español, aunque este otro docente pone en funcionamiento una estrategia contraria a la suya, como se explica a continuación 


\section{El caso del profesor de español}

El docente de español prefiere no marcar demasiado los pasos de los alumnos ya que espera que estos inventen y que se esfuercen para cumplir la tarea. Valora mucho la autonomía de sus estudiantes, pero, si lo necesitan, les proporciona apoyo sobre la marcha, como se observa en el Fragmento 4:

Fragmento 4:

$59 \quad M M M \quad Y$ que hay: maneras de preguntar $\uparrow$ según como preguntes $\uparrow$ la

60 formulación es más dificil $\uparrow$ entonces(.) cambiando un poco la forma $\downarrow$

Le parece apropiado que todas las cuestiones de la didáctica se centren en su propia persona y en su relación con cada alumno y por ello tiene una actitud abierta y está preparado para todas las situaciones imprevistas que puedan surgir en el aula. Da valor a su gran capacidad de observación e identificación de los fenómenos de su enseñanza, de sus alumnos y de su aprendizaje. Cree que debe actuar con el máximo respeto hacia la personalidad de sus alumnos ("si tienes 24 alumnos, que son 24 mundos") y rechaza todo lo que los pudiera reducir a meros objetos de los que se pueda llegar a obtener solo sus resultados concretos y visibles de la enseñanza impartida. Y aunque se lleve muy bien con sus alumnos, él también mantiene alto su estatus, lo que se manifiesta en su control sobre todo el proceso de aprendizaje. Parece que no da mucha importancia a que la evaluación sea una herramienta útil para sus alumnos y por tanto tampoco a los medios concretos que acompañan a la misma, como la nota o el bonus.

Este docente cree que es más útil trabajar en la adaptación al nivel del alumno y ajustar tanto el material como las instrucciones propias y los comentarios para que el aprendizaje pueda producirse, como se observa en el Fragmento 5:

Fragmento 5:

\begin{tabular}{|c|c|}
\hline$M M M$ & a parte de la motivación es que: para que todo esto esté \\
\hline 86 & permeable para ellos $\uparrow$ para que ellos lleguen a estos niveles $\downarrow$ (.) \\
\hline 87 & es decir(.) e:dificultad $\uparrow$ dificultarles el: \\
\hline 88 & rendimiento lingüístico $\downarrow$ (.) cuando son \\
\hline & que no están aún en su Norte $\uparrow($.) es chocar contra la pared un poco(.) No $\uparrow$ \\
\hline
\end{tabular}

Siendo él mismo un gran observador, espera de sus alumnos que aprendan observando y fijándose tanto en él, como profesor, como en los compañeros que destacan con sus estudios. Sin embargo no menciona si comunica todo lo que ha observado a sus alumnos en el 
momento de la evaluación. Respeta a sus alumnos y sus particularidades debidas a la diversidad, aunque no la atribuye solamente al plurilingüismo, pero a la vez considera que dicha diversidad es la fuente principal de las dificultades a las que deben afrontarse los docentes.

\section{El caso de los profesores de catalán}

A los docentes de la asignatura de catalán también les parece importante su adaptación a las necesidades actuales de los grupos o de los cursos y a las diferentes edades. Además, prestan mucha atención a la actitud de sus alumnos y aparte de favorecer a los motivados no creen que puedan influir en los otros, que les parecen unos "vagos", citando la palabra utilizada en la línea 455 del Fragmento 6. Consideran sobreprotegidos a los alumnos del colegio, pero no especifican de qué manera. En la didáctica de los docentes de catalán la clave es la motivación de los alumnos como se observa en el Fragmento 6:

Fragmento 6:

$\begin{array}{ll}453 \text { JJJ } & \text { una de las } \\ 454 & \text { conclusiones que sacamos } \uparrow(.) \text { es que } \downarrow \text { (..) el que es vago } \uparrow(. .) \\ 455 & \text { es vago } \uparrow(.) \text { aunque pueda elegir el tipo de trabajo } \downarrow \text { que quiera } \downarrow \text { (..) y:. } \\ 456 & \text { aunque } \downarrow \text { y... el chico } \downarrow(.) \text { que es trabajado:r } \uparrow(. .) \text { a: la: que ve una } \\ 457 & \text { cosa que le motiva un poco } \downarrow \text { (.) y que el } \downarrow \text { (.) puede:. hacerlo un } \\ 458 & \text { poco mejo:r } \uparrow(.) \text { se crece } \uparrow\end{array}$

Prefieren echar mano de todo tipo de medios para conseguir la colaboración activa de los mismos. Intentan motivar también a través de una gran diversidad de posibles trabajos o tareas que ofrecen a los alumnos para su elección.

Les parece útil el uso de los bonus "lo que hace, es compensar" a los alumnos L2 por su esfuerzo añadido motivándolos a no verse limitados por el miedo a cometer errores, ni en sus trabajos escritos ni en su participación oral en las clases.

Asimismo, subrayan la importancia de los pequeños, pero regulares, trabajos en el aprendizaje de estos alumnos, como se observa en el Fragmento 7:

Fragmento 7:

\begin{tabular}{|c|c|}
\hline 385 JJJ & entonces con este sistema $\uparrow$ de: de muchos más mini:test (.) \\
\hline 386 & evidentemente pues no le pondrás un $1 \uparrow($ ) \\
\hline 387 & porque le faltará $\uparrow($.$) la parte de :$ participación en clase $\downarrow$ pero sí que \\
\hline 388 & puede conseguir un $2+\uparrow($.$) porque ha sacado: buena nota \downarrow$ \\
\hline
\end{tabular}


Creen incluso que sustituir la nota oral por la de estos pequeños trabajos escritos ayuda a aprender mejor la gramática o la ortografía y de este modo alcanzar mejores notas en sus exámenes finales, a los que corresponden el $60 \%$ de la nota final. De este modo producen muchas notas que ofrecen informaciones tanto para el alumno como para el docente y pueden equilibrar (40\%) las notas finales de los exámenes (60\%), además estas notas motivan a los alumnos ya que el éxito es asequible para todos, y, por último, estos múltiples pequeños trabajos sirven de preparación para los pocos exámenes finales.

Ambos docentes parece que excluyan prácticamente la evaluación de la participación oral. Sin embargo, los trabajos orales también pueden sustituir a exámenes finales, así que la oralidad no desaparece, ocurre solamente que los trabajos escritos están sobrerrepresentados en las notas. Una cierta sobrerrepresentación se ve también en la mayor ponderación dada a la gramática y al estilo comparado con la otorgada al contenido.

Sin embargo, definen a un cierto grupo de alumnos, a los que llaman "tímidos", que representan un reto para ellos por ser poco comunicativos, y destacan de ellos su activa colaboración en los proyectos escritos. Esto se puede interpretar como que la comunicación oral o la participación en las clases sí tienen un peso en su didáctica, ya que en el caso del bonus también subrayaron su importancia como estímulo de la participación de los alumnos, y solamente prefieren excluir el rendimiento oral de la evaluación, pero no queda claro el motivo.

Plasman animadamente las pautas fundamentales del colegio en cuanto a la evaluación que han adaptado a sus fines, pero sin embargo no hablan de aspectos más concretos o detallados de la evaluación que les parece útil aplicar con alumnos de estas características.

\section{Conclusiones}

Los sistemas de evaluación de cada profesor demuestran que consideran como parte integral la evaluación de su proceso de enseñanza, aunque la respuesta al reto de los alumnos plurilingües hizo que cada uno, según sus creencias, conocimientos y propia tradición escolar haya creado una aplicación propia que, utilizando las categorías de Hufeisen y Marx (2007), son más bien complementarias, ya que los docentes pueden ejercer influencia en la tradición de aprendizaje y los factores emocionales cognitivos del plurilingüismo. En nuestro caso, los docentes de catalán y español trabajan más fuertemente el factor emocional, y el de alemán trabaja más explícitamente las estrategias y herramientas de evaluación, aportando los valores de su tradición de enseñanza, sus saberes y sus creencias. En este centro, pues, los docentes se 
complementan uno al otro y consiguen que los alumnos se conviertan realmente en hablantes plurilingües con competencia intercultural, en vez de hablantes monolingües múltiples.

El plurilingüismo de los alumnos es un reto para todos al que responden con sistemas de evaluación complejos y diversos desde el punto de vista pedagógico. El planteamiento es monolingüe y ninguno de los docentes cree que más allá de los baremos de la evaluación debiera haber más colaboración entre los departamentos. Tampoco mencionan el término del plurilingüismo, sino que hablan de la diversidad a la que ven como un reto o algo que les genera dificultades, y no expresan en ningún momento los aspectos positivos de los alumnos plurilingües. No citan ninguna de las ventajas que se atribuyen a los hablantes plurilingües: tener su propio proceso de gramática espontanea (Meißner, 2004), desarrollar competencias transversales (Guasch, 2014) o poseer una mayor capacidad de llevar a cabo actividades de reflexión metalingüística (Bialystok, 2009).

Aunque adopten la metodología del enfoque por proyectos, hablando de la evaluación están justificando la evaluación de los productos finales y no en el proceso mismo. Según su explicación, la nota oral debe incluir ciertos aspectos del mismo, sin embargo, no se llega a tener información específica. Como subrayaron, entro otros, Lorenzo, Trujillo y Vez (2011) y García y Kleyn (2013) la enseñanza de los plurilingües en última instancia depende de la calidad educativa de los docentes. En este trabajo hemos observado que los docentes están preparados y motivados, pero gestionan por separado, aunque con éxito, la formación de alumnos que acaban siendo plurilingües. Es razonable pensar que, con una mayor concienciación sobre el plurilingüismo y la evaluación formativa, acompañada de una mayor colaboración entre los departamentos, podrían obtener aún mejores resultados.

\section{Bibliografía}

Bedore, L., \& Peña, E. (2008). Assessment of bilingual children for identification of language impairment: Current findings and implications for practice. International Journal of Bilingual Education and Bilingualism, 1-21.

Bialystok, E. (2009). Bilingualism: The good, the bad, and the indifferent. Bilingualism: Language and Cognition, 12(1), 3-11.

Birello, M., \& Sánchez-Quintana, N. (2013). Conceptualizaciones sobre las lenguas y creencias sobre el plurilingüismo de los docentes a través de sus relatos de vida lingüística. RILA, 2-3, 191-205.

Björklund, S. (2005). Toward trilingual education in Vaasa/Vasa. International Journal of the Sociology of Language, 23-40.

Blommaert, J., \& Backus, A. (2013). Superdiverse repertoires and the individual. En I. de Saint Georges, J.-J. Weber, \& U. o. Luxembourg (Eds.), Multilingualism and Multimodality, Vol. 2 (págs. 11-33). Rotterdam: Sense Publishers. 
Cambra, M. (2000). Recerca i formació en didáctica de la llengua . En A. Camps i Mundó, I. Ríos García; M. Cambra i Giné (Coords.) Recerca i formació en didáctica de la llengua (161-172). Barcelona: Graó.

Cambra, M. (2011). Plurilingüsme i ensenyament de llengües. Articles de la lengua i de la Literatura, 53, 77-84.

Cambra, M., \& Palou, J. (2007). Creencias, representaciones y saberes de los profesores de lenguas en las nuevas situaciones plurilingües escolares de Cataluña. Cultura $y$ Educación, 19(2), 149-163.

Camps, A. (2003). Secuencias didácticas para aprender a escribir. Barcelona: Graó.

Camps, A., Guasch, O., Milian, M., \& Ribas, T. (2005). Bases per a l'esenyament de la gramàtica. Barcelona: Graó.

Casanova, M. (1999). Manual de evaluación educativa. Barcelona: La Muralla.

Cassany, D., \& Sanz, G. (1998). Enseñar lengua. Barcelona: Graó.

Cassany, D., Luna, M., \& Sanz, G. (2002). Enseñar lengua. Barcelona: Graó.

Cenoz, J. (2003). The additive effect of bilinguism on third language acquisition. A review. International Journal of Bilinguism, 7, 71-88.

Cenoz, J., \& Genesee, F. (1998). Beyond bilingualism: Multilingualism and multilingual education. Clevedon: Multilingual Matters.

Cros, A., \& Vilá, M. (1997). La llengua oral: Propostas per a l'avaluació. En T. Ribas, L'avaluació formativa en l'àrea de llengua (187-209). Barcelona: Graó.

Fives, H. B. (2012). Spring cleaning for the "messy" construct of teachers' beliefs. En APA educational psychology handbook: Volume 2 (págs. 471-499). Washington: K.R. Harris, S. Graham, \& T. Urdan.

Galambos, S. J., \& Goldin-Meadow, S. (1990). The effect of learning two languages on levels of metalingustic awareness. Cognition, 34, 1-56.

García, O., \& Kleyn, T. (2013). Teacher education for multilingual education. In C. A. Chapelle (Ed.) The encyclopedia of applied linguistics (págs. 5543-5548). Oxford, UK: Wiley-Blackwell.

Guasch, O. (2014). La reflexión interlingüística en la enseñanza y el aprendizaje de las lenguas. Revista textos. Didáctica de la Lengua y de la Literatura, 64, 26-36.

Herdina, P., \& Jessner, U. (2002). A Dynamic Model of Multilingualism. Perspectives of Change in Psycholinguistics. Clevedon: Cromwell Press Ltd.

Herdina, P., \& Jessner, U. (2002). A dynamic model of multilingualism: Perspectives of change in psycholinguistics. Clevedon: Multilingual Matters.

Hufeisen, B., \& Marx, N. (2007). How can DaFnE and EuroComGerm contribute to the concept of receptive multilingualism? Theoretical and practical considerations. En J. Thije, \& L. Zeevaert (Coords.), Receptive multilingualism: Linguistic analyses, language policies and didactic concepts (págs. 307-321). Amsterdam: John Benjamins.

Hufeisen, B., \& Neuner, G. (2001). Mehrsprachigkeitskonzept - Tertiärsprachlernen Deutsch nach Englisch. Strasbourg: Council of Europe Publishing.

Jessner, U. (2008). Teaching third languages: Findings, trends and challenges. Cambridge University Press, 15-56.

Kecskés, I., \& Papp, T. (2000). Foreign language and mother tongue. Mahwah, NJ: Lawrence Erlbaum.

Kress, G. (2013). Recognizing learning: A perspective from a social semiotic theory of multimodality. En I. de Saint Georges \& J. J. Weber (Eds.), Multilingualism and multimodality. Current challenges for educational studies (págs. 119-141). Rotterdam: Sense Publishers. 
Lorente, P. (2011). Retos de la evaluación en Lengua Castellana y Literatura para el siglo XXI. Tejuelo, 11, 104-127.

Lorenzo, F. T. (2011). Educación bilingüe. Integración de contenidos y segundas lenguas. Madrid: Síntesis.

Lüdi, G. (2000). Multilingual repertoires and the consequences. http://www.dylanproject.org/Dylan_en/presentation/dissemination/articles/assets/Unibas-LuediMultilingualRepertoriesAndTheConsequencesForLinguistic Theory.pdf

Lüdi, G., \& Py, B. (2009). To be or not to be. International Journal of Multilingualism, 6,(2,), $154-167$.

Luise, M. C. (2013). Plurilinguismo e multilinguismo in Europa per una Educazione plurilingue e interculturale. $L E A($ n.2), 525-535.

Meissner, F.-J. (2004). Transfer und Transferieren: Anleitungen zum Interkoprehensionsunterricht. Aachen: Klein y Rutke.

Neuner, G. (2001). The concept of plurilingualism and tertiary language didactics. En B. \&. Hufeisen (Ed.), Mehrsprachigkeitskonzept - Tertiärsprachlernen - Deutsch nach Englisch (pp. 13-35). Strasbourg: Council of Europe Publishing.

Noguerol, A. (2001). Cómo tratar la diversidad lingüística y cultural. En M. Casas, \& C. Tomas (Coords.) Educación Primaria. Orientaciones y recursos (6-12 años) (228309). Barcelona: Cisspraxis.

Nussbaum, L. (2014). Una didàctica sociolingüística de les llengües? Bellaterra Journal of Teaching \& Learning Language \& Literature, 1-13.

Palou, J. (2008). L'Ensenyament i l'aprenentatge del català com a primera llengua a l'escola. Creences $i$ actuacions dels mestres amb relació a les activitats de llengua oral a l'etapa primària. Barcelona: Institut d'Estudis Catalans.

Phipps, S., \& Borg, S. (2009). Exploring tensions between teachers' grammar teaching beliefs and practice. System, 37, 380-390.

Ribas, T. (1997). L' avaluació formativa e el procés d' escriptura: un instrument per a l' aprenentatge. En T. Ribas (Coord.) L'avaluació formativa en l'àrea de llengua. (págs. 131-156). Barcelona: Graó.

Ribas, T. (2010). La evaluación en el área lingüística. Textos de Didáctica de la Lengua y la Literatura (10-21). Barcelona: Graó.

Rothweiler, M., \& Ruberg, T. (2011). Der Erwerb des Deutschen bei Kindern mit nichtdeutscher Erstsprache. München: Deutsches Jugendisnstitut e. V.

Sandín Esteban, M. P. (2003). Investigación cualitativa en educación. Fundamentos y tradiciones. Madrid: McGraw and Hill Interamericana.

Voet Cornelli, B., Schultz, P., \& Tracy, R. (2013). Sprachentwicklungsdiagnostik bei Mehrsprachigkeit. Monatsschr Kinderheilkunde, 161, 911-917.

Welling, A. (1998). Mehrsprachigkeit und Sprachheilpedagogik - die verhängnisvolle Geschichte einer Pathologisierung. En G. S. J., Über Mehrsprachigkeit (págs. 21-42). Tübingen: Narr.

Woods, D. (1996). Teacher Cognition in language teaching. Beliefs, decision-making and classroom practice. Cambridge, MA: Cambridge University Press.

\footnotetext{
${ }^{\mathrm{i}}$ La transcripción sigue la ortografía convencional. Se respetan las pausas que hace la persona que habla. No se han utilizado signos de puntuación convencionales. No se corrigen las palabras, ni las construcciones gramaticales incorrectas desde el punto de vista de la normativa lingüística. Las palabras de código diferente se escriben en negrita. No se indican los fenómenos relacionados con los aspectos fonéticos. Se numeran las líneas.
} 


\section{ii Aspectos prosódicos: \\ $\uparrow$ Tono ascendente \\ $\downarrow$ Tono descendente \\ $::$ Alargamiento de un sonido \\ Subr Énfasis}

(.) Pausa corta (El número de puntos indican la duración de la pausa)

iii Reciben un bonus en la nota de los exámenes y se les aplica un baremo especial de corrección de faltas en los

exámenes de redacción o comentario, en los que además se les reduce el número de palabras que deben producir.

\section{Anexo}

En cada entrevista semiestructurada fueron formuladas las mismas preguntas sobre la evaluación:

1. ¿Te ha generado dificultades el método de evaluación de este centro por sus diferencias respecto al sistema alemán/español/catalán?

2. ¿Existe una coordinación entre los diferentes departamentos de lengua en cuanto a la evaluación?

3. ¿ ¿Funcion a bien el sistema del bonus?

4. ¿Qué hay detrás de la ponderación del $60 \%$ de la nota escrita y $40 \%$ para la oral?

5. ¿Qué trabajos se evalúan durante el curso?

6. ¿ ¿Se evalúan sólo los productos finales o también los subproductos de una actividad?

7. ¿Tienen los alumnos una evaluación externa?

8. ¿Se utiliza una evaluación normativa o una evaluación criterial?

9. ¿Se miden los resultados del aprendizaje o la evolución?

10. ¿Se adapta la evaluación a la clase o a los alumnos?

11. ¿Se dialoga la evaluación con los alumnos?

12. ¿Se miden los resultados del aprendizaje o la evolución del alumno?

13. ¿Se utilizan andamiajes?

14. ¿Se utilizan rúbricas o baremos de asignación de puntos?

15. ¿Es flexible el plan de estudios respecto a los resultados de los trabajos?

16. ¿Se utiliza la coevaluación y la autoevaluación de los alumnos?

17. ¿Cómo se reacciona a la dependencia de lo literal en los trabajos?

\section{Biodata:}

Zita Mate es licenciada en filología por la Universidad ELTE de Budapest y en Traducción e Interpretación por la Universitat Autònoma de Barcelona donde también finalizó sus estudios de Máster de Didáctica de Lengua y Literatura en 2015.

\section{E-mail: matezita48@gmail.com}

Para citar este artículo:

Mate, Z. (2017). Diseño y fiabilidad de un cuestionario sobre la comprensión auditiva/audiovisual. Bellaterra Journal of Teaching \& Learning Language \& Literature, 10(3), 28-46. DOI: http://doi.org/10.5565/rev/jt13.724

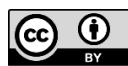

